Document downloaded from:

http://hdl.handle.net/10251/179818

This paper must be cited as:

Zennaro, M.; Pelsser, C.; Albinet, F.; Manzoni, P. (2020). Evaluating the performance of NRENs in deploying loT in Africa: the case for TTN. IEEE. 1-4.

https://doi.org/10.1109/CCNC46108.2020.9045213

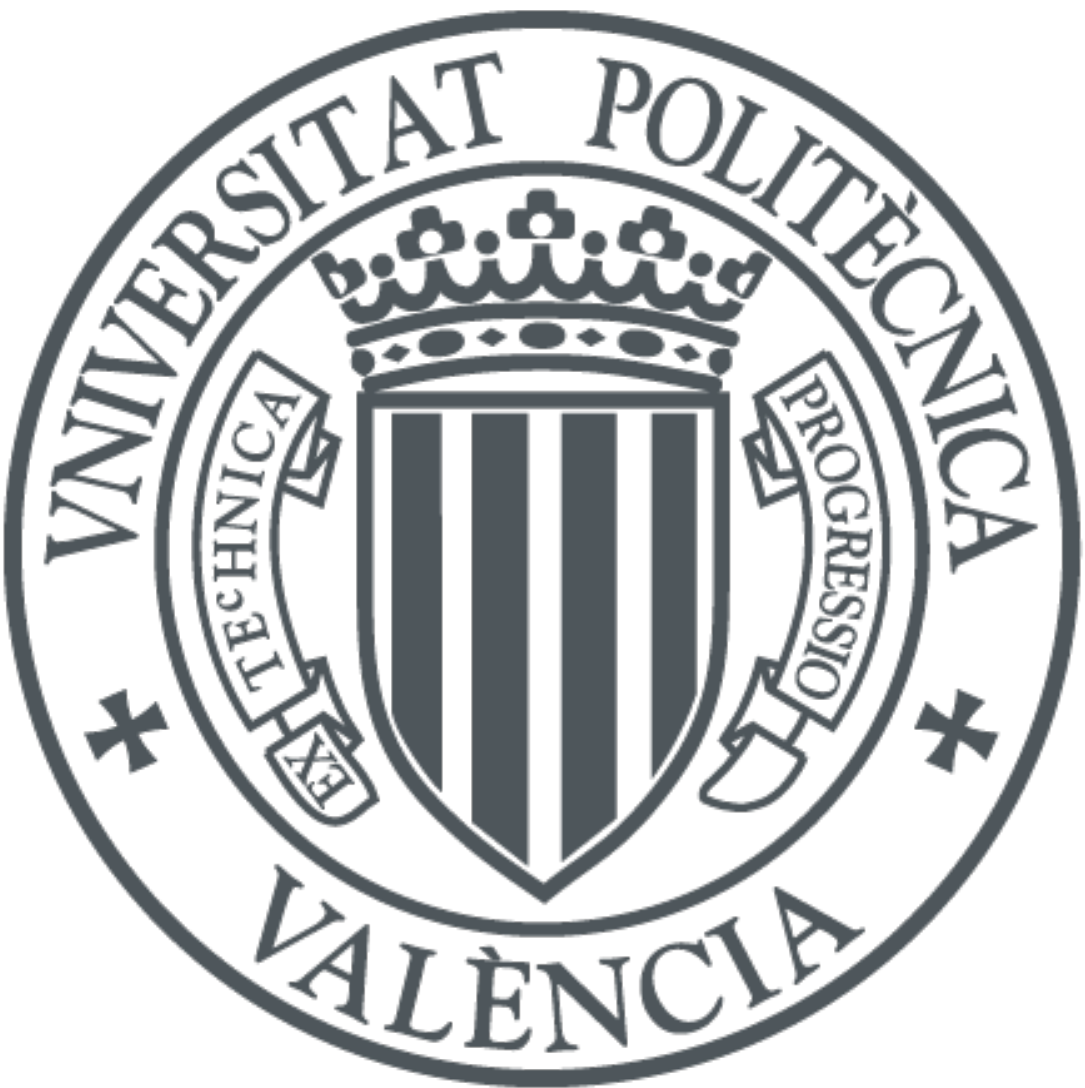

The final publication is available at

https://doi.org/10.1109/CCNC46108.2020.9045213

Copyright IEEE

Additional Information 


\section{Evaluating the performance of NRENs in deploying IoT in Africa: the case for TTN}

\author{
Marco Zennaro \\ ICTP \\ Trieste, Italy \\ mzennaro@ictp.it
}

\author{
Cristel Pelsser \\ University of Strasbourg \\ Strasbourg, France \\ pelsser@unistra.fr
}

\author{
Franck Albinet \\ Independent Consultant \\ Guéthary, France \\ franckalbinet@gmail.com
}

\author{
Pietro Manzoni \\ Universitat Politécnica de Valéncia \\ Valencia, Spain \\ pmanzoni@disca.upv.es
}

\begin{abstract}
The growth of the Internet worldwide has been fueled by the development of NRENs, networks of academic and educational institutions. In Africa the establishment of NRENs is more recent. In this paper we analyze the readiness of African NRENs to be part of "The Things Network" (TTN), a network of IoT gateways that has fostered the growth of IoT in Europe by adopting a community network model. We analyse RTT and packet loss toward the nearest TTN network server, in African countries where RIPE Atlas probes are hosted both in academic and commercial networks. Our conclusion is that NRENs and commercial ISPs are on an equal foot in hosting TTN gateways in most countries we considered.

Index Terms-
\end{abstract}

\section{INTRODUCTION}

The Things Network (TTN) is an initiative to build a worldwide open source infrastructure to facilitate a public Internet of Things (IoT) [1]. TTN is based on LoRaWAN, a Low-Power Wide-Area Network (LPWAN) protocol that can be used to build IoT networks. LoRaWAN provides low-bandwidth communications over long distances. In typical applications IoT nodes send few bytes of data every few minutes/hours over distances in the range of 1-10 kilometers. These technical characteristics allow the use of cheap devices that can last five to ten years on a single battery. TTN operates by allowing users to share the access to gateways, which play the role of access points for LoRaWAN networks. As the amount of traffic generated by nodes is very limited, users are willing to share the access to their gateways and this allows the network to have a greater coverage. The cost of LoRaWAN gateways is not the largest problem when setting up a new local IoT network. Finding the locations to install the gateways is the biggest obstacle. This is where NRENs can play an important role as they have locations spread all over cities.

There are now over TTN 300 communities worldwide, in more than 100 cities. TTN offers an opportunity to bring academic communities and businesses together by creating an open ecosystem.

\section{National Research AND EdUCATION Networks}

A National Research and Education Network (NREN) provides the data communications and more in general the eservices needs of the research and education community of a given country. NRENs are being deployed to address the specific needs in terms of bandwidth, quality of service, security, and reliability of the Research and Education communities. These needs cannot typically be met by commercial providers since they would require high costs with possibly low economical returns.

These types of network are becoming really helpful in tackling the digital divide between academic and research institutions in different geographical areas. They provide the possibility to train academic staff, to attract young scientists which the country needs for stimulating the national research agenda. NRENs also offer the possibility to participate in international projects and therefore to access to state-of theart facilities particularly in some domains where there are development potentials.

NRENs Community involves Universities, Research and Development Institutions, Primary and Secondary Schools, Libraries, Museums, Hospitals, Telecom Service Providers and Internet Exchange Points (IXPs). The Major Global NREN Clusters are:

- GÉANT2, a high-bandwidth, academic Internet network serving Europes research and education community. It connects more than 50 million users. GÉANT2 is cofunded by the European Commission and Europes national research and education networks. It is at the core of global research networking and links to other worldwide REN clusters around the globe on a continental level. These include TEIN3 and Internet2 described below.

- TEIN3 is the third generation of the Trans-Eurasia Information Network (TEIN). It connects the research and educational communities in 11 countries across the AsiaPacific region with a dedicated high-capacity internet network and provides them with direct connectivity to Europes GÉANT2 cluster. TEIN3 enables global cooperation and gives millions of users across the AsiaPacific region access to global knowledge and joint research projects.

- Internet2 was founded in 1996. It provides the backbone that connects universities, K-12 schools, government agencies, regional and state education networks in the US ${ }^{1}$. Internet 2 serves 317 U.S. universities, 60 government agencies, 43 regional and state education networks.

\footnotetext{
${ }^{1}$ https://www.internet2.edu/about-us/
} 
The first step for the establishment of NRENs in Africa [2] can be tracked back to the deployment of undersea fiber optic cables along the East and West coasts of Africa in the last decade, improving Internet connectivity in several countries. These developments highlighted the need for African countries to establish their own NRENs and to have them connected as a continental wide network through an initiative called the Africa Research and Education Network (AfREN). In the last decade, regional associations of NRENs have been formed to promote the AfREN concept; UbuntuNet Alliance in Eastern and Southern Africa, and WACREN in Western and Central Africa.

The European Union (EU) provided the financial means to kick-start this initiative by funding the AfricaConnect project on a cost share basis with partner African countries [3]. The EU had already been funding a program to connect North African NRENs to Europe in a project called EUMEDCONNECT. At the time of that project a group of countries in the Middle East and North Africa formed an association of NRENs called ASREN. The aim of these two projects and similar EU-funded ones in Asia, Central Asia, and Latin America was to enable the emerging NRENs in the regions to establish linkages regionally and to connect to GEANT [4], the European Research and Education network backbone, and through it to connect to all other NRENs of the world.

The current development of all African NRENs, including those in North Africa, is now largely planned around this partnership of the three regional associations, namely UbuntuNet, WACREN and ASREN, and their European partners, and timed around the funding cycle of the AfricaConnect project, as phase one has concluded and AfricaConnect2 has began in July 2015. The interest in establishing NRENs has developed in almost all countries in Africa. However, growth is varied with just eighteen networks in operation as of June 2016; four in ASREN, three in WACREN and eleven in the UbuntuNet Alliance. Within some of those networks the average bandwidth provided to member institutions can be low with an average of 100 Mbps.

\section{LORAWAN TECHNOLOGY}

LoRaWAN is a comprehensive network architecture that details a Low Power, Wide Area (LPWA) networking technology. It was designed to interconnect battery operated "things" and provides a bi-directional communication service with end-toend security features. The architecture is based on a star-ofstars topology in which specific devices called gateways relay messages between end-devices and a central network server. The wireless communication takes place through a single-hop link between the end-device and one or many gateways.

The specification defines the device-to-infrastructure physical layer parameters (LoRa) and the specific LoRaWAN protocol to allow for full interoperability among manufacturers. It defines the technical implementation but it does not define any commercial model or type of deployment, e.g., public, shared, private, enterprise. This characteristics offers the industry the freedom to innovate and differentiate how it is used. The

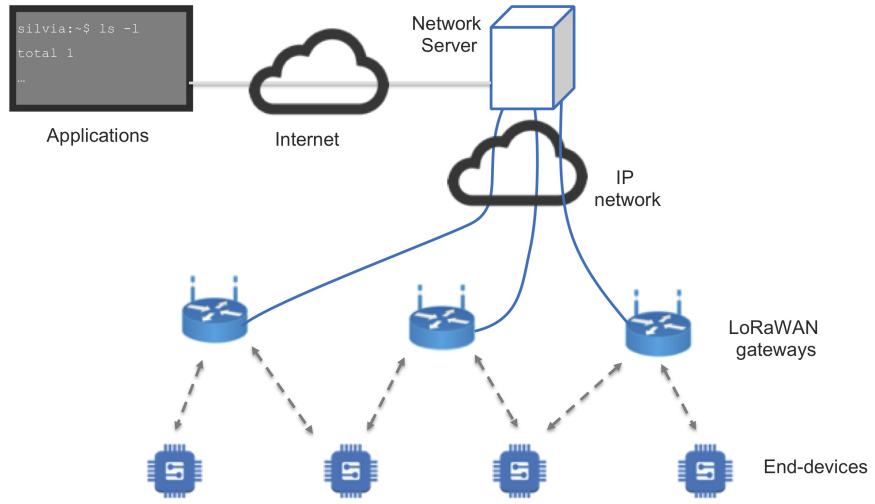

Fig. 1. The LoRaWAN architecture.

LoRaWAN specification is developed and maintained by the LoRa Alliance ${ }^{2}$, an open association of collaborating members. With LoRa and LoRaWAN the intention was to provide the possibility to deploy Internet of Things applications fast in areas where large distances are involved, yet low bandwidth is needed. Low bandwidth makes it ideal for practical IoT deployments with less data or where data transmissions are not constant, for example with waste management where the information that is required is whether a waste bin is full or not. Moreover, the end-to-end delay (or the RTT) can be relatively high, with values below one second being acceptable [5].

Conversely, packet loss is a more crucial factor. In LoRaWAN, once a message has been delivered, there is no acknowledgement of receipt. However, nodes can request acknowledgements. In this case, if many gateways receive the same packet, the cloud has to choose one gateway to respond at a fixed time, usually a couple of seconds later. The problem is that when a gateway is transmitting back to the node, it stops listening to everything else. So, if an application needs a lot of acknowledgements, the gateway will very likely spend more time transmitting acknowledgements than listening, which will eventually lead to a network saturation. Therefore, classical applications do not require acknowledgments and that's why having a reliable network with very small packet loss rate is crucial if we don't want to lose relevant information.

\section{Ripe AtLas MEASUREMENTS}

The RIPE Atlas infrastructure is composed of probes and anchors. Both sets of devices are able to perform measurements. Anchors take the form of racked servers in their hardware version or virtual machines (VMs). At the time of writing VMs represent a minority of the anchors. On the other hand, probes are USB-powered, lightweight devices.

There are around 10k probes ${ }^{3}$ and 400 anchors $^{4}$ in the RIPE Atlas infrastructure. While probes and anchors are mostly deployed in Europe and North America, there are a total of

\footnotetext{
${ }^{2}$ https://lora-alliance.org/

${ }^{3} 10398$ connected probes on July 1st, 2019.

${ }^{4} 406$ anchors as of July 3rd 2019.
} 


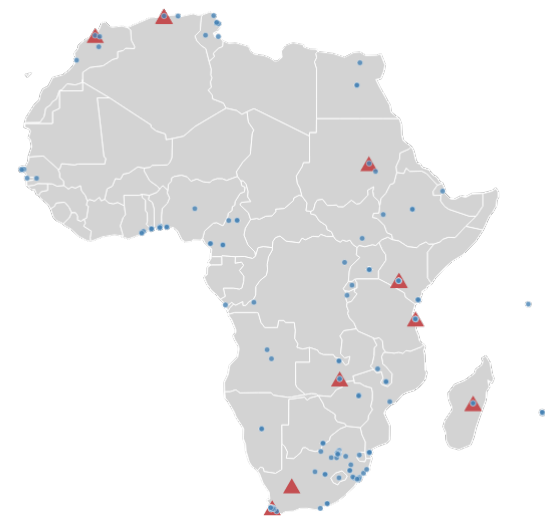

Fig. 2. NREN (red triangles) and non-NRENs (blue circles) probes.

238 probes on the African continent ${ }^{5}$ hosted in 35 countries and 136 Autonomous Systems [6]. An Autonomous System (AS) is a group of IP networks run by one or more network operators with a single, clearly defined routing policy.

The RIPE Atlas probes can be scheduled to perform ping, traceroute, DNS, SSL/TLS and HTTP measurements to userdefined destinations. Users can specify the frequency of the measurements, the duration of the experiment, the number of packets to be sent, the flow-id in order to explore all paths or pin the measurement to some paths, and whether or not they desire a DNS resolution prior to the measurement. The RIPE Atlas infrastructure also performs what is called "builtin" measurements. The built-ins are defined by the RIPE Atlas team. They run from all probes and from all anchors to other probes, anchors and other sets of destinations. They probe the DNS root servers for delay measurements, for example, to provide information on world connectivity to the DNS roots. The results of built-in measurements are made public and downloadable on the RIPE Atlas website as are most userdefined measurements.

\section{AtLas-TTN measurements Results}

Thanks to RIPE's support we were able to schedule an experiment to measure the RTT from 216 probes in Africa to the TTN Network Server in Ireland every $15 \mathrm{~min}$. This TTN Network server is used by African TTN nodes as there is no server in the continent. The same network server is used by European TTN users. The experiment started in November 2018 and gathered data up to July 2019. Figure 2 shows the locations of Atlas probes in Africa involved in this experiment.

In this preliminary analysis, we focused on data collected in December 2018 in African countries where both NRENs and non-NRENs probes could be identified as we wanted to compare TTN access from academic and commercial connections.

The identification of NRENs probes was done on a case by case basis using metadata scattered across various resources such as:

\footnotetext{
${ }^{5}$ https://atlas.ripe.net/results/maps/density/
}

- RIPE Atlas probes API;

- Wikipedia article on National research and education network $^{6}$;

- WHOIS directory, BGPView.io ${ }^{7}$ and IPInfo.io ${ }^{8}$ services.

The manual task described above allowed to identify probes belonging to the following NRENs:

- Algerian Academic Research Network;

- Kenya Education Network;

- Moroccan Academic and Research Wide Area Network (MARWAN);

- Research and Education Network for Academic and Learning Activities (iRenala), Madagascar;

- Mozambique Research and Education Network (MoRENet);

- Sudanese Research and Education Network;

- Tanzania Education And Research Network;

- Tertiary Education and Research Network (Tenet), South Africa and;

- Zambia Research and Education Network (ZAMREN).

Of all the probes hosted in Africa we could only use the data coming from 8 countries where we were able to identify probes in both academic and commercial networks. About 350,000 Traceroute commands were run in the month of December 2018 in the 8 countries of interest. Figure 3 shows the RTTs, per country and NREN membership, computed using the mean value of RTTs coming from the probes, where each measurement consisted of three packets. In Figure 4 we show the Packet loss per country and NREN membership, in the 8 countries under consideration. We observe that the RTTs are often similar from NRENs and non-NRENs with a few exceptions such as Kenya. On the other hand, packet loss is often higher in non-NRENs except for Malaga.

To ensure the Reproducibility of our research and to disseminate the techniques and tools used for the analysis, a companion Python Jupyter Notebook containing the whole data analysis pipeline is available publicly ${ }^{9}$.

\section{CONCLUSIONS}

In this paper, we singled out RIPE Atlas probes hosted in NRENS from the ones with a commercial Internet access. We showed that this process is mostly a manual task and insist on the need to tag measurement infrastructure with accurate metadata. We then observed that the RTT is not significantly different between the 2 classes of providers, academic versus commercial, in most countries. In the eight countries under consideration the RTT values would not impact the access to TTN. Packet loss is not significantly high and is not distinctive between NRENs and commercial ISPs for most countries and both classes of networks seem to converge to similar performances. In two of the eight countries, however, the packet loss is significantly higher in commercial networks compared to academic ones.

\footnotetext{
${ }^{6} \mathrm{https} / / /$ en.wikipedia.org/wiki/National_research_and_education_network ${ }^{7}$ https://bgpview.io

${ }^{8}$ https://ipinfo.io/

${ }^{9}$ http://tiny.cc/cy $16 \mathrm{cz}$
} 

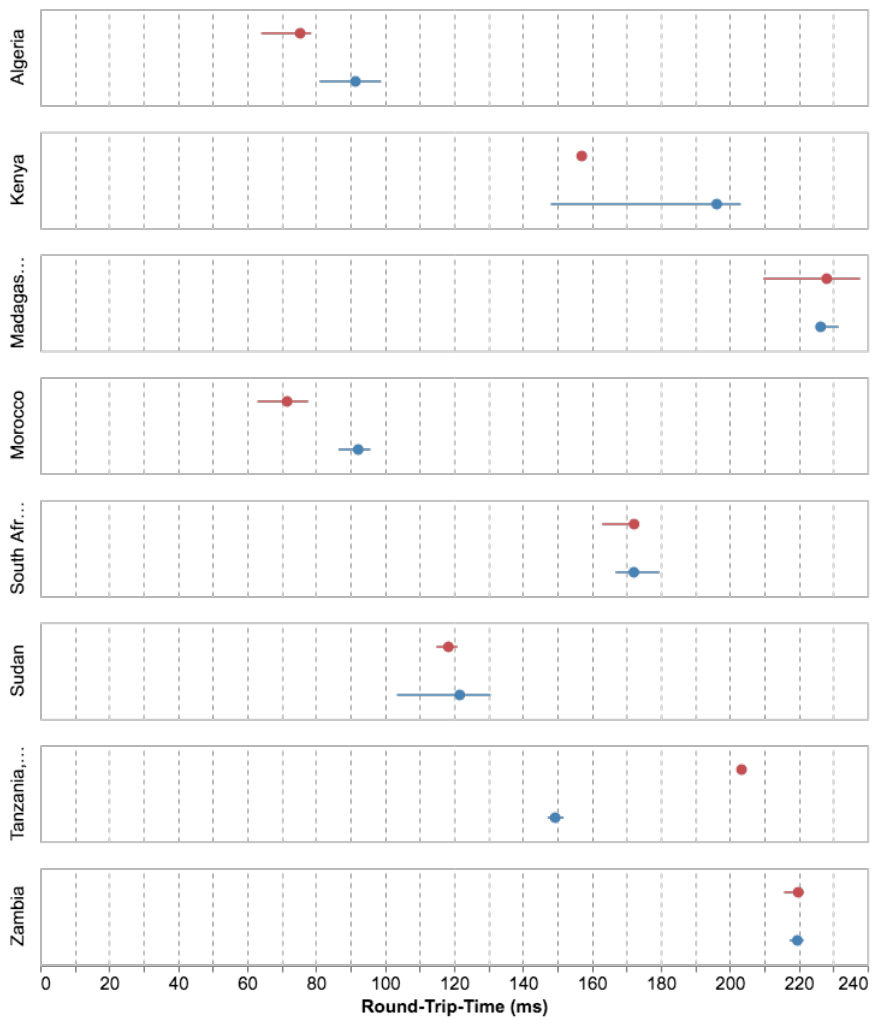

Fig. 3. RTTs median (circle), first and third quartiles (bars) for NREN (red) and non-NREN (blue) probes.
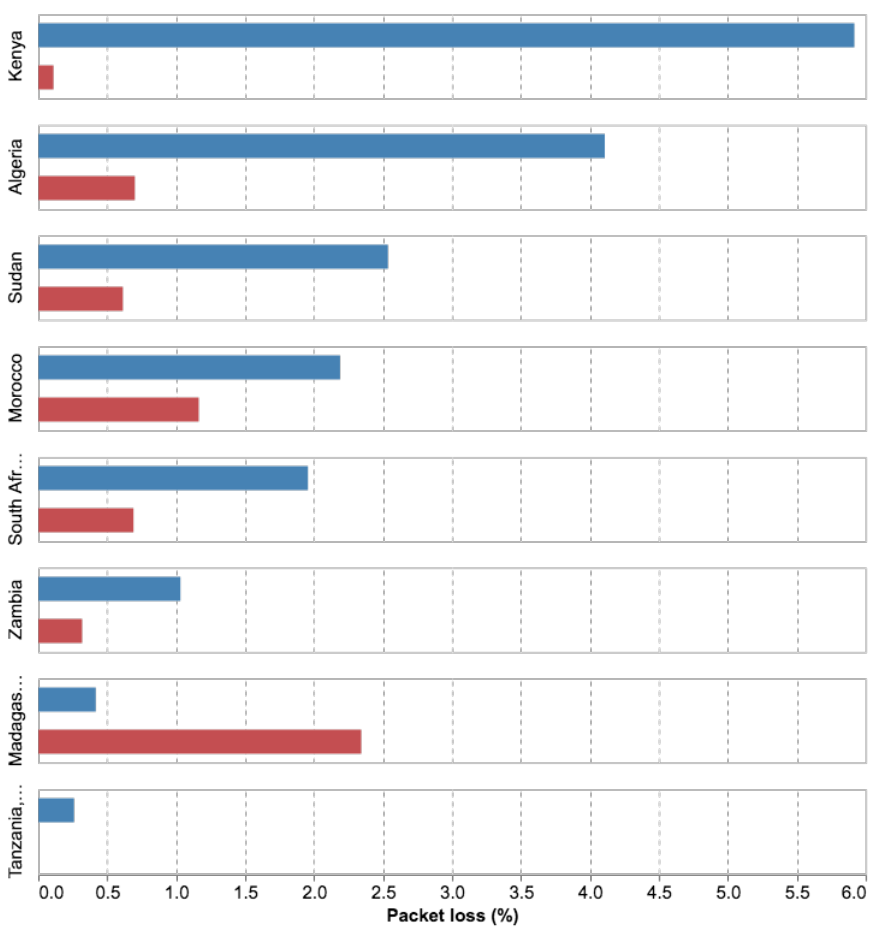

Fig. 4. Packet loss for NREN (red) and non-NREN (blue) probes.
We note the low number of RIPE Atlas probes on the African continent. This has an impact on the generality of our findings. To draw conclusions with a higher confidence we need more sources in Autonomous Systems both commercial and academic. The current study relies on RIPE Atlas built-in measurements. We did not generate additional measurements. In the future, we plan to measure at higher rates in order to determine losses with higher accuracy.

We suggest to add tags to probes in measurement infrastructures in order to ease the studies such as this one. RIPE Atlas already indicates the hosting country, the latitude and longitude of probes, as well as their access type (IPv4 and/or IPv6). We suggest information on the type of provider, commercial or academic. If the probe is connected to a commercial ISP, a label with its rank in the Internet hierarchy would also be helpful. Most researchers rely on CAIDA AS ranking data ${ }^{10}$ set for this purpose. An integration of this information would help reproduce past studies and run them longitudinally. A tag indicating if the probe is connected to a network serving IoT gateways would also be very helpful.

\section{ACKNOWLEDGEMENT}

This work was partially supported by the "Ministerio de Ciencia, Innovación y Universidades, Programa Estatal de Investigación, Desarrollo e Innovación Orientada a los Retos de la Sociedad, Proyectos I+D+I 2018", Spain, under Grant RTI2018-096384-B-I00.

We want to thank Johan Ter Beest and Alvaro Vives of the RIPE NCC for their support and help in setting up the experiment.

\section{REFERENCES}

[1] P. A. Barro, M. Zennaro, and E. Pietrosemoli, "Tltn the local things network: on the design of a lorawan gateway with autonomous servers for disconnected communities," in 2019 Wireless Days (WD), April 2019, pp. 1-4.

[2] R. Sanby, H. Suleman, and J. Chavula, "Efficient topology discovery for african NRENs," in 2016 IST-Africa Week Conference, May 2016, pp. $1-8$.

[3] M. Foley, "The role and status of national research and education networks (NRENs) in africa," World Bank Education, Technology \& Innovation: SABER-ICT Technical Paper Series (n. 05). Washington, DC: The World Bank, Available at: http://saber.worldbank.org, 2016.

[4] A. Hankel, H. Astsatryan, and W. Narsisian, "The GEANT green team: An example of how organizations can use a community approach to promote the use of ICT in sustainability efforts," in Computer Science and Information Technologies (CSIT), Sep. 2015, pp. 125-127.

[5] N. Blenn and F. A. Kuipers, "LoRaWAN in the wild: Measurements from The Things Network," CoRR, vol. abs/1706.03086, 2017. [Online]. Available: http://arxiv.org/abs/1706.03086

[6] R. Fanou, F. Valera, P. Francois, and A. Dhamdhere, "Reshaping the african internet: From scattered islands to a connected continent," Computer Communications, vol. 113, pp. 25-42, Nov. 2017. 